\title{
Prevention of mother-to-child transmission: the key of hepatitis B virus elimination
}

\author{
Chih-Lin $\operatorname{Lin}^{1,2} \cdot$ Jia-Horng Kao $3,4,5,6,7$
}

Received: 19 February 2018 / Accepted: 27 March 2018/Published online: 4 April 2018

(C) Asian Pacific Association for the Study of the Liver 2018

More than half a century after the initial discovery, hepatitis B virus (HBV) is one of the most common viral infections in humans. With a global heterogeneous prevalence, the HBV epidemic remains a monumental threat of health. In Asia, most HBV patients acquire the virus perinatally or in early childhood and develop a life-long chronic infection [1]. The mechanisms of vertical HBV transmission include intrauterine infection and transmission during delivery [2]. For example, the prevalence of chronic HBV infection in the general population ranged 11-20\% in Taiwan during 1980s [3]. Approximately, 40\% of $\mathrm{HBV}$ carriers were born to $\mathrm{HBsAg}$-positive mothers and 85-95\% of newborns with HBV exposure consequently had chronic infection [4]. Thus, mother-to-child transmission (MTCT) during the perinatal period is the major transmission route of HBV in hyperendemic areas [1]. Prevention of MTCT is thus the most important strategy to reduce the global burden of $\mathrm{HBV}$ infection.

Immunoprophylaxis with the combination of hepatitis B immunoglobulin and hepatitis $B$ vaccine in newborns has been documented to prevent perinatal HBV transmission.

Jia-Horng Kao

kaojh@ntu.edu.tw

1 Department of Gastroenterology, Renai Branch, Taipei City Hospital, Taipei, Taiwan

2 Department of Psychology, National Chengchi University, Taipei, Taiwan

3 Graduate Institute of Clinical Medicine, National Taiwan University, College of Medicine, 1 Chang-Te St., Taipei 10002, Taiwan

4 Department of Internal Medicine, National Taiwan University Hospital, Taipei, Taiwan

5 Hepatitis Research Center, National Taiwan University, National Taiwan University Hospital, Taipei, Taiwan

6 Department of Medical Research, National Taiwan University, National Taiwan University Hospital, Taipei, Taiwan

7 Ministry of Education, Executive Yuan, Taipei, Taiwan
Currently, universal infant HBV vaccination has been implemented in 180 countries worldwide [5]. In Taiwan, the first country to launch a nationwide universal hepatitis B vaccination program [6], the HBV exposure rate (antiHBc positivity) decreased from 38 to $4.6 \%$ in children after implementation of universal hepatitis B immunization [7]. Of particular note is that the HBV carriage rate in infants and children remarkably declined up to $90 \%$ in 2012 [8]. Similarly, the prevalence of HBV infection in children was also reduced in different parts of the world after successful implementation of universal hepatitis B immunization [9]. In addition to preventing HBV infection of infants, immunoprophylaxis implementation also reduces hepatocellular carcinoma (HCC) in children, teenagers and young adults $[10,11]$. In Taiwan, the incidence of HCC in children decreased from 0.92 per 100,000 in the unvaccinated cohort to 0.23 per 100,000 in the vaccinated birth cohorts [11].

These encouraging data provide the convincing evidence that prevention of HBV infection is the primary strategy for global HBV eradication. However, the immunoprophylaxis failure due to HBV carrier mothers with a high viral load is a great challenge. A cohort study from Taiwan revealed that an increased risk of MTCT was correlated with a high maternal viral load [12]. To enhance the blocking rate of MTCT, antiviral therapy with nucleos(t)ide analogs (NAs) in pregnancy should be considered.

In this issue, He et al. presented the safety and efficacy of lamivudine or telbivudine treatment initiated at the first trimester of pregnancy in mothers with a high viral load [13]. The authors retrospectively enrolled 94 highly viremic mothers, and $49(52.1 \%)$ of them initiated lamivudine or telbivudine in the first trimester of pregnancy. At postpartum week 28, the MTCT rate was significantly reduced in the mothers with NAs treatment than those without $(0 / 61$ or $0 \%$ vs $4 / 34$ or $11.76 \%, p=0.028$ ). Of note, the birth defect rates of infants were similar between the treatment group and the control group. These findings indicated that 
Fig. 1 A proposed algorithm of prophylactic antiviral therapy for pregnant $\mathrm{HBs} A g$-positive female to prevent mother-tochild transmission (MTCT) of HBV. Maternal serum HBV DNA level of $20,000 \mathrm{IU} / \mathrm{ml}$ as the threshold of low and high maternal viral load. All infants born to $\mathrm{HBsAg}$-positive female should also receive

immunoprophylaxis of hepatitis $B$ vaccination with or without hepatitis B immunoglobulin after birth. $H B V$ hepatitis B virus, $H B s A g$ hepatitis B surface antigen, $H B e A g$ hepatitis $\mathrm{B}$ e antigen

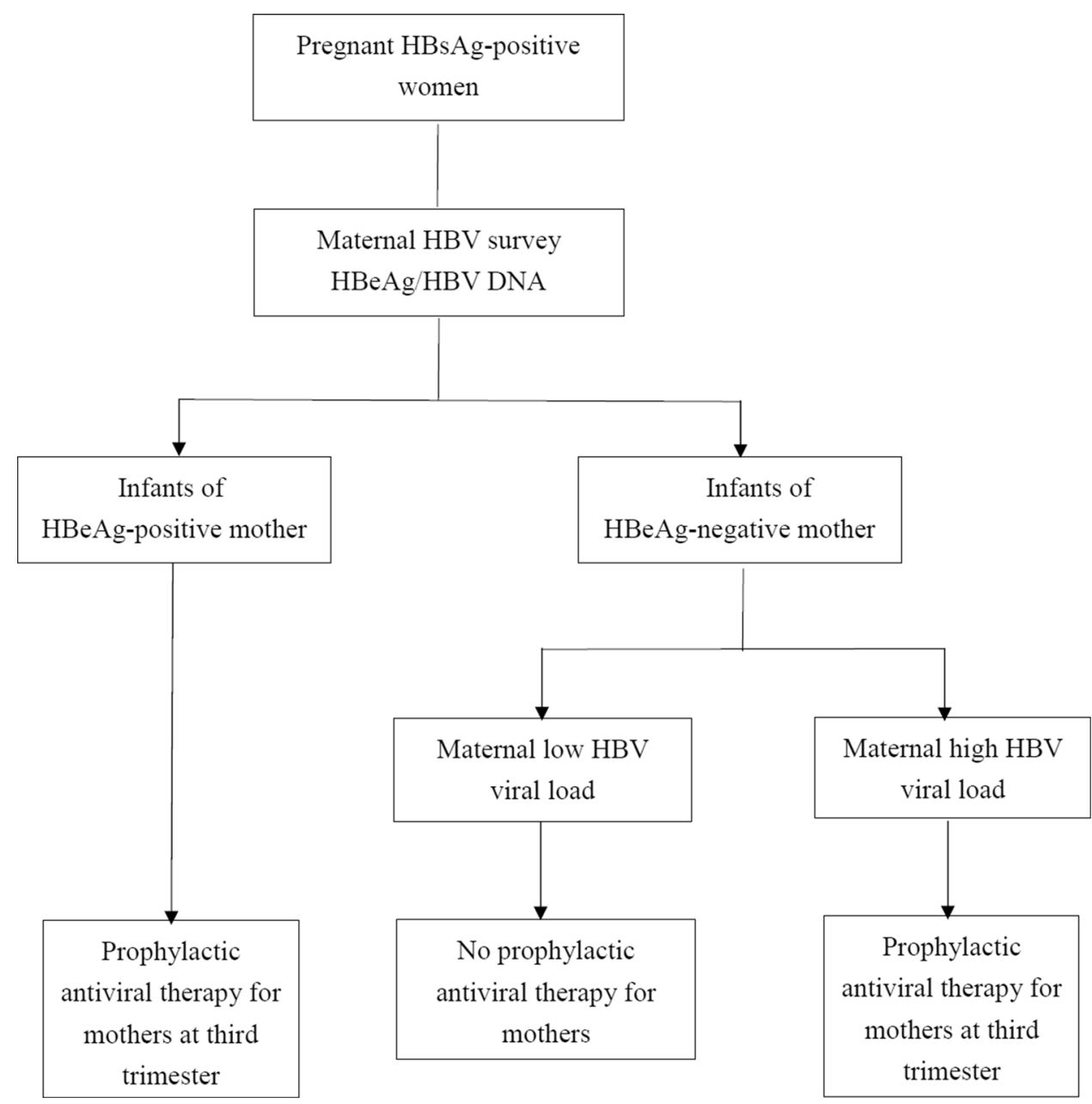

of gestation until 1 month postpartum in highly viremic HBV mothers. Compared to the mothers without antiviral prophylaxis, the mothers who received TDF had a significantly lower rate of infant HBsAg positivity $[15,16]$. The safety profiles of infants, including birth defect and prematurity, were comparable between mothers with and without TDF prophylaxis. These findings were further confirmed in a meta-analysis, which demonstrated that any antiviral therapy in the third trimester of pregnancy has profound HBV suppression to reduce MTCT in mothers with a high viral load [17]. Because none of the NAs has been approved for use in pregnancy, the safe profiles for NAs initiated in first trimester pregnancy should be cautious and deserve more studies.

The final issue is to define the indication of initiating treatment of $\mathrm{HBV}$ in pregnant women. In a retrospective study of 2356 children, the offspring of $\mathrm{HBeAg}$-positive mothers had significant higher risk for chronic $\mathrm{HBV}$ infection than those of HBeAg-negative mothers (9.26 vs. $0.23 \%, p<0.001$ ) [18]. A prospective study from Taiwan further demonstrated that maternal viral load was significantly associated with the risk of HBV transmission. The 
estimated predictive rate of MTCT at maternal viral load level of $5 \log _{10}$ copies/ml was $0.9 \%$. Surprisingly, the rates of MTCT increased sharply for every log increase in maternal viral load [12]. Similarly, a recent study of 1177 mother-infant pairs also indicated that higher maternal viral loads were associated with a higher risk of MTCT [19]. In the current study, the mothers in the immune tolerant phase of CHB were excluded [13]. The characteristics of immune tolerant phase entail $\mathrm{HBeAg}$ positivity and very high serum HBV DNA levels, thus these females have the highest risk of MTCT. Therefore, all infants born to HBsAg-positive female should also receive immunoprophylaxis of hepatitis B vaccination with or without hepatitis B immunoglobulin after birth. Furthermore, maternal serum HBV DNA level of $5 \log _{10}$ copies/ml (20,000 IU/ $\mathrm{ml}$ ) may serve as an indication for prophylactic antiviral therapy to prevent MTCT, irrespective of HBeAg status (Fig. 1).

In summary, the combination of immunoprophylaxis in newborns and antiviral prophylaxis for mothers with a high viral load is plausible to completely interrupt MTCT of HBV [20]. In addition, existing potent NAs have remarkably improved the disease control of chronic hepatitis B. With the complete interruption of MTCT and a successful functional HBV cure, the mission of $\mathrm{HBV}$ elimination would be achievable by 2030 [21].

\section{Compliance with ethical standards}

Conflict of interest Chih-Lin Lin and Jia-Horng Kao have no conflict of interest.

Ethical approval This article does not contain any studies with human participants or animals performed by any of the authors.

\section{References}

1. Kao JH, Chen DS. Global control of hepatitis B virus infection. Lancet Infect Dis. 2002;2:395-403

2. Mavilia MG, Wu GY. Mechanisms and prevention of vertical transmission in chronic viral hepatitis. J Clin Transl Hepatol. 2017;5:119-129

3. Lin CL, Kao JH. Perspectives and control of hepatitis B virus infection in Taiwan. J Formos Med Assoc. 2015;114:901-909

4. Stevens CE, Toy P, Kamili S, et al. Eradicating hepatitis B virus: the critical role of preventing perinatal transmission. Biologicals 2017;50:3-19
5. WHO. Guidelines for the prevention, care and treatment of persons with chronic hepatitis B infection. WHO Publishers; 2015. http://www.who.int/hiv/topics/hepatitis/en/. Accessed $19 \mathrm{Feb}$ 2018

6. Chen DS, Hsu NHM, Sung JL, et al. A mass vaccination program in Taiwan against hepatitis B virus infection in infants of hepatitis B surface antigen-carrier mothers. JAMA. 1987;257:2597-2603.

7. Ni YH, Huang LM, Chang MH, et al. Two decades of universal hepatitis B vaccination in Taiwan: impact and implication for future strategies. Gastroenterology. 2007;132:1287-1293

8. Ni YH, Chang MH, Wu JF, Hsu HY, Chen HL, Chen DS. Minimization of hepatitis B infection by a 25-year universal vaccination program. J Hepatol. 2012;57:730-735

9. Kao JH. Hepatitis B vaccination and prevention of hepatocellular carcinoma. Best Pract Res Clin Gastroenterol. 2015;29:907-917

10. Chang MH, You SL, Chen CJ, et al. Decreased incidence of hepatocellular carcinoma in hepatitis B vaccinees: a 20-year follow-up study. J Natl Cancer Inst 2009;101:1348-1355

11. Chang MH, You SL, Chen CJ, et al. Taiwan Hepatoma Study Group. Long-term effects of hepatitis B immunization of infants in preventing liver cancer. Gastroenterology. 2016;151:472-480

12. Wen WH, Chang MH, Zhao LL, et al. Mother-to-infant transmission of hepatitis B virus infection: significance of maternal viral load and strategies for intervention. J Hepatol. 2013;59:24-30

13. He T, Bai Y, Cai H, et al. Safety and efficacy of lamivudine or telbivudine started in early pregnancy for mothers with active chronic hepatitis B. Hepatol Int. 2018. https://doi.org/10.1007/ s12072-017-9839-5

14. Jourdain G, Ngo-Giang-Huong N, Harrison L, et al. Tenofovir versus placebo to prevent perinatal transmission of hepatitis B. N Engl J Med. 2018;378:911-993

15. Chen HL, Lee CN, Chang CH, et al. Taiwan Study Group for the Prevention of Mother-to-Infant Transmission of HBV (PreMIT study). Efficacy of maternal tenofovir disoproxil fumarate in interrupting mother-to-infant transmission of hepatitis B virus. Hepatology. 2015;62:375-386

16. Pan CQ, Duan Z, Dai E, et al. China Study Group for the Motherto-Child Transmission of Hepatitis B. Tenofovir to prevent hepatitis B transmission in mothers with high viral load. N Engl J Med. 2016;374:2324-2334

17. Brown RS Jr, McMahon BJ, Lok AS, et al. Antiviral therapy in chronic hepatitis B viral infection during pregnancy: a systematic review and meta-analysis. Hepatology. 2016;63:319-333

18. Chen HL, Lin LH, Hu FC, et al. Effects of maternal screening and universal immunization to prevent mother-to-infant transmission of HBV. Gastroenterology. 2012;142:e772

19. Lu Y, Zhu FC, Liu JX, et al. The maternal viral threshold for antiviral prophylaxis of perinatal hepatitis B virus transmission in settings with limited resources: a large prospective cohort study in China. Vaccine. 2017;35(48 Pt B):6627-6633

20. Chen HL, Wen WH, Chang MH. Management of pregnant women and children: focusing on preventing mother-to-infant transmission. J Infect Dis. 2017;216(suppl_8):S785-S791

21. Tseng TC, Kao JH. Elimination of hepatitis B: is it a mission possible? BMC Med. 2017;15:53 